\title{
Mathematical modeling of Eulerian currents induced by wind and waves at the sea surface
}

\author{
M. Rahman ${ }^{1}$ \& D. Bhatta ${ }^{2}$ \\ ${ }^{1}$ Faculty of Computer Science, Dalhousie University, Canada \\ ${ }^{2}$ The University of Texas Pan-American, USA
}

\begin{abstract}
This paper deals with the study of mathematical modeling of Eulerian currents in ocean circulations. The wave-wave interaction of four progressive waves traveling with four wave numbers and four frequencies are elegantly described by Komen et al. (J. Phys. Oceanogra., 14 (1984), 1271-1285). Therefore, detailed investigations are avoided in this present paper. We shall rather devote our study to the description of the analytic solutions of the Eulerian currents present in the ocean circulation. This study contains the mathematical descriptions of nonlinear wave interactions, wind and wave induced surface currents, unsteady Eulerian currents in one-dimension, and steady two-dimensional Eulerian currents in ocean circulations. A variety of solutions that satisfy the governing equations with their initial and boundary conditions are obtained. A Laplace transform method in conjunction with the convolution concept is used as a solution technique and the accuracy of the solution is confirmed by using the powerful separation of variables method. Some of the solutions are graphically illustrated in non-dimensional forms and the physical meaning is described.

Keywords: Eulerian currents, Lagrangian currents, Ekman spirals, mathematical modeling, ocean circulations, surface currents, nonlinear waves, wave energy, wind stress, steady currents, unsteady currents, Laplace transforms, integral transforms, convolution, separation of variables method.
\end{abstract}

\section{Introduction}

The ocean current is defined as a continuous, directed movement of ocean water generated by the forces acting upon this mean flow, such as breaking waves, wind, Coriolis effect, temperature and salinity differences and tides caused by the 
gravitational pull of the Moon and the Sun. The current's direction and strength are influenced by the depth of the ocean, the shoreline configurations and interaction with other currents. A deep current is any ocean current at a depth of greater than 100 meters. Ocean currents can flow for great distances, and together they create the great flow of the global conveyor belt which plays a dominant part in determining the climate of many of the Earth's regions. Perhaps the most striking example is the Gulf Stream, which makes northwest Europe much more temperate than any other region at the same latitude.

In oceanography and in fluid dynamics in general, our observations can be made in two ways: Lagrangian measurements and Eulerian measurements. Lagrangian measurements involve following a parcel of fluid as it moves. For example, we could measure temperatures from a weather balloon or from a free floating buoy. In other way to make the measurements is to have an observation site geographically fixed. For example, we can measure temperature at a fixed weather station or from an anchored buoy in the ocean. Measurements made in this manner are known as Eulerian measurements. These descriptions can very easily be extended to encompass the current measurements in ocean circulations. Eulerian currents play a very important role in the study of ice-floe drift in ocean circulations. So we shall concentrate our study in the mathematical modeling of Eulerian currents in ocean circulations.

Measurements of ocean current are collected using a variety of methods. One popular way to measure ocean currents is to determine the water's velocity at one fixed place in the ocean. This type of measurement is called Eulerian, in honor of the Swiss mathematician Leonhard Euler. This is typically accomplished using an electro-mechanical current meter. Surface ocean currents are generally winddriven and develop their typical clockwise spirals in the northern hemisphere and counter-clockwise rotation in the southern hemisphere because of the imposed wind stress. In wind-driven currents, the Ekman [2] spiral effect results in the currents flowing at an angle to the driving wind. The areas of surface ocean currents move somewhat with the season; this is most notable in equatorial currents. Due to physical importance of Eulerian currents we present here their fundamental aspects using sophisticated mathematical models.

\section{Mathematical formulation of wave-induced surface currents}

In this section we shall first formulate the equations of the Eulerian current in twodimensions. Given wind field $U_{10}$ at 10 meters reference height, the wave balance equation can be written as (see Longuet-Higgins [3])

$$
\frac{d E(f, \theta)}{d t}=\left(S_{i n}-S_{d s}\right)\left(1-f_{i}\right)+S_{n l}+S_{i c e},
$$

where $\frac{d}{d t}=\left(\frac{\partial}{\partial t}+\mathbf{C}_{g} \cdot \nabla\right)$ denotes total differentiation when traveling with the group velocity. Here $E(f, \theta)$ is the two-dimensional wave spectrum which is a function of frequency $f$, (cycles/sec, Hertz), and direction $\theta$, time $t$ and position $\mathbf{x}$, and where $\mathbf{C}_{g}$ is the group velocity, and $\nabla$ is a gradient operator. Also we define 
that $S_{i n}$ is the input of energy due to wind, $S_{d s}$ is the energy dissipation due to white capping, $S_{n \ell}$ is the nonlinear transfer between spectral components due to wave-wave interactions, $S_{i c e}$ is the change in energy due to wave interactions with ice floes, and $f_{i}$ is the fraction of the area of the ocean covered by ice.

The accepted formulation for wind input $S_{i n}$, as parameterized in the WAM model of Hasselmann [4], Hasselmann [5] and Hasselmann et al. [6], suggests that $S_{\text {in }}$ should be represented by

$$
S_{i n}=\beta \omega E(f, \theta)
$$

where $\beta$ is a non-dimensional function of sea state maturity and $\omega=2 \pi f$ represents the angular (radial) frequency, which is related to the wave number $k$ through the deep water dispersion relation $\omega^{2}=g k$ for deep ocean and $\omega^{2}=g k$ tanh $k h$ for finite depth ocean. Description of all other parameters can be found in the work of Rahman [7], and so will not be repeated here.

The associated Stokes drift is the mean velocity following a fluid particle, and therefore, by definition, is a Lagrangian property. Let us consider that $\mathbf{U}_{s}$ is the Lagrangian velocity of a particle at initial position $(\mathbf{x}, c, t=0)$, and the Stokes drift at time $t$ is simply given by

$$
\mathbf{U}_{s}(\mathbf{x}, t)=4 \pi \iint f \mathbf{K} e^{2 k c} E(f, \theta) d f d \theta
$$

following Jenkins [8]. The vertical Lagrangian coordinate $c$ corresponds to the usual vertical Eulerian coordinate $z$ at the initial time $t=0$. The quasi-Eulerian current $\mathbf{U}_{E}$ satisfies the following partial differential equation as described by Jenkins [8]:

$$
\begin{aligned}
\frac{\partial \mathbf{U}_{E}}{\partial t}+\mathbf{f} \times \mathbf{U}_{E}= & \frac{\partial}{\partial c}\left(\nu \frac{\partial \mathbf{U}_{E}}{\partial c}\right)-\mathbf{f} \times \mathbf{U}_{s} \\
& -2 \pi \int d f \int f \mathbf{K} S_{d s} 2 k N e^{2 k c} d \theta
\end{aligned}
$$

where ice floes are not assumed to be present. The quasi-Eulerian current $\mathbf{U}_{E}$ can be thought of as being the Eulerian mean current $\mathbf{U}_{e}$ with reference to a Lagrangian coordinate system and so $\mathbf{U}_{E}=\mathbf{U}_{L}-\mathbf{U}_{s}=\mathbf{U}_{e}$, where $\mathbf{U}_{L}$ is the Lagrangian mean current. Other variables of (4) are $\nu$, the eddy viscosity and $\mathbf{f}=(0,0, \lambda)$, the Coriolis acceleration $|\mathbf{f}|=2 \Omega \sin \phi$, where $\Omega$ is the Earth's angular velocity and $\phi$ is the latitude. The vector $\mathbf{K}$ is defined as $\mathbf{K}=\left(\cos \left(\frac{\pi}{2}-\right.\right.$ $\theta), \sin \left(\frac{\pi}{2}-\theta\right)$, which is related to wave number $k$ by $\mathbf{k}=k \mathbf{K}=k[\sin \theta, \cos \theta]$. The integral representation of (4) represents the generation of $\mathbf{U}_{E}$ from the waves through the wave dispersion $S_{d s}$. The coefficient $N$ represents the momentum transfer from waves to current, and it can be assumed that $N=1$. Finally, the partial derivative term $\frac{\partial}{\partial c}\left(\nu \frac{\partial \mathbf{U}_{E}}{\partial c}\right)$ represents the vertical transport of momentum 
by viscous shear stress. The boundary condition at the sea surface is

$$
\left.\nu \frac{\partial \mathbf{U}_{E}}{\partial c}\right|_{c=0}=\frac{\vec{\tau}}{\rho_{w}}-2 \pi \int d f \int f \mathbf{K} S_{i n} d \theta
$$

where $\tau=|\vec{\tau}|=\sqrt{\tau_{x}^{2}+\tau_{y}^{2}}=\rho_{a} C_{D}\left|U_{10}^{2}\right|$ is the wind stress on the water surface , $\rho_{a}$ is the air density, $\rho_{w}$ is the water density, and $C_{D}$ is the drag coefficient. In two dimensions, $\mathbf{U}_{e}=(u, v, 0)$, where $u$ and $v$ are the horizontal components of the Eulerian currents in $x-y$ plane. Also we define Stokes drift components in two-dimensions as $\mathbf{U}_{\mathbf{s}}=\left(u_{s}, v_{s}, 0\right)$.

We now avoid detailed description of the development of other terms leading to the final equations of the Eulerian currents because of the page limitation. Interested reader is referred to the works of Hasselmann [5], Jenkins [8], Komen et al. [9] and Perrie and $\mathrm{Hu}$ [10]. We write the governing equations of the Eulerian currents explicitly with their boundary conditions and initial conditions as follows (considering usual vertical coordinate $z$ ):

$$
\begin{aligned}
& \frac{\partial u}{\partial t}-\lambda v=\frac{\partial}{\partial z}\left(\nu \frac{\partial u}{\partial z}\right)+\lambda v_{s}-S_{d x} \\
& \frac{\partial v}{\partial t}+\lambda u=\frac{\partial}{\partial z}\left(\nu \frac{\partial v}{\partial z}\right)-\lambda u_{s}-S_{d y}
\end{aligned}
$$

The boundary conditions can be written as:

\section{Surface boundary conditions:}

$$
\text { at } \begin{aligned}
z=0: \quad \nu \frac{\partial u}{\partial z} & =\frac{\tau_{x}}{\rho_{w}}-S_{x w} \\
\nu \frac{\partial v}{\partial z} & =\frac{\tau_{y}}{\rho_{w}}-S_{y w} .
\end{aligned}
$$

\section{Bottom boundary conditions:}

$$
\text { at } z=\left\{\begin{array}{cc}
-\infty \text { (infinite depth); } & u=0 \\
-h \text { (finite depth); } & v=0
\end{array}\right\}
$$

The initial conditions are assumed to be:

$$
\text { at } t=0: \quad u=0 ; \quad v=0 .
$$

Throughout this investigation, we shall assume that $u$ and $v$ are functions of the vertical coordinate $z$ and the time $t$. And they do not depend upon the horizontal coordinates $x$ and $y$. We shall start out our investigation with a very simple problem of Eulerian currents in ocean circulation. 


\section{Unsteady Eulerian current in one-dimension}

This section deals with the unsteady Eulerian current in one-dimension. We discuss here the simple analytical solutions with simple boundary conditions and initial condition for the cases of deep water, finite depth water and shallow water. The method of Laplace transforms (together with the separation of variables method) are employed in obtaining the solutions. The solutions are described below.

\section{Case I: Deep ocean}

We shall consider first the deep ocean Eulerian current in one dimension. In this case, the Coriolis force does not play any part. We assume that the wave dispersion term is negligible. We also assume that the wave input term is negligible and the eddy viscosity $\nu$ is constant. Equation (6) with its boundary conditions (7) and (8), and initial condition (9) can be written is a simple form as follows:

$$
\begin{aligned}
\frac{\partial u}{\partial t} & =\nu \frac{\partial^{2} u}{\partial z^{2}} \\
z=0: \quad \frac{\partial u}{\partial z} & =\frac{\tau_{x}}{\nu \rho_{w}} \\
z=-\infty: \quad u & =0 \\
\text { and } t=0: \quad u & =0 .
\end{aligned}
$$

\section{Solution}

The Laplace transform method will be suitable for this mathematical model. We define the Laplace transform of $u(z, t)$ as $\mathcal{L}(u(z, t))=\int_{0}^{\infty} u(z, t) e^{-s t} d t$ such that $\mathcal{L}\left\{\frac{\partial u}{\partial t}\right\}=s \mathcal{L}\{u(z, t)\}$. Equations (10) through (12) can be transformed as follows:

$$
\begin{aligned}
\frac{d^{2}}{d z^{2}}(\mathcal{L}\{u\})-\left(\frac{s}{\nu}\right)(\mathcal{L}\{u\}) & =0 \\
z=0: \frac{d}{d z}(\mathcal{L}\{u\}) & =\frac{\tau_{x}}{\nu \rho_{w}}\left(\frac{1}{s}\right) \\
z=-\infty: \mathcal{L}\{u\} & =0 .
\end{aligned}
$$

Using the transformed boundary conditions we obtain the solution as follows:

$$
\mathcal{L}\{u\}=\left(\frac{\tau_{x}}{\rho_{w} \sqrt{\nu}}\right)\left\{\frac{\exp (\sqrt{s / \nu} z)}{s \sqrt{s}}\right\}
$$


The Laplace inverse formula gives the solution as follows (see Abramowitz and Stegun [11]):

$$
u(z, t)=\left(\frac{\tau_{x}}{\rho_{w} \sqrt{\nu}}\right)\left\{2 \sqrt{\frac{t}{\pi}} \exp \left(-\frac{z^{2}}{4 \nu t}\right)+\left(\frac{z}{\sqrt{\nu}}\right) \operatorname{erfc}\left(-\frac{z}{2 \sqrt{\nu t}}\right)\right\} .
$$

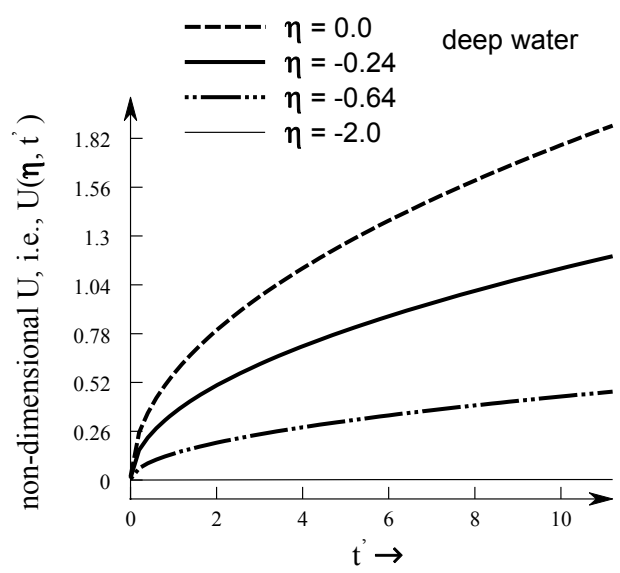

Figure 1: Eulerian currents in deep water at various depth.

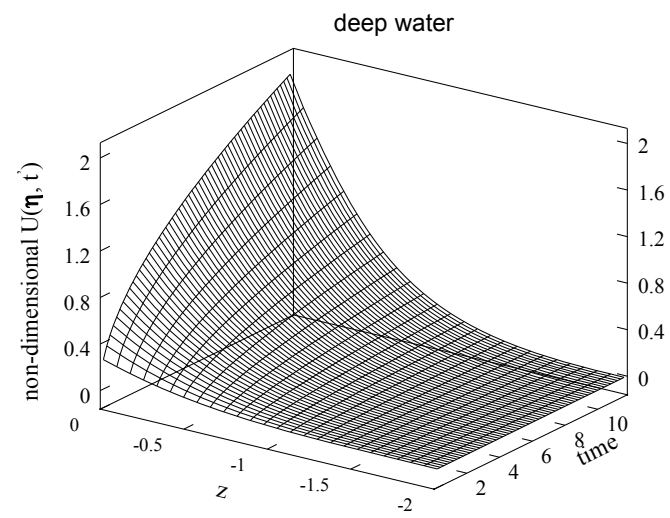

Figure 2: Eulerian currents in deep water as a function of $z$ and $t$.

If we assume the non-dimensional variables as $\eta=\frac{z}{2 \sqrt{\nu t}}$ which happens to be the similarity variable, and the non-dimensional time as $t^{\prime}=4 \nu t / L^{2}$ where $L$ is 
some typical length (may be wavelength), then we can write the equation (18) in non-dimensional form as follows:

$$
U\left(\eta, t^{\prime}\right)=\sqrt{t^{\prime}}\left[\frac{1}{\sqrt{\pi}} \exp \left(-\eta^{2}\right)+\eta \times \operatorname{erfc}(-\eta)\right] .
$$

where

$$
U\left(\eta, t^{\prime}\right)=u(z, t) /\left(\frac{L \tau_{x}}{\rho_{w} \nu}\right),
$$

and the range of $\eta$ is $-\infty<\eta \leq 0$ and $t^{\prime}>0$.

At the sea surface $z=0$ (i.e. in non-dimensional variable $\eta=0$ ), the Eulerian current is given by

$$
u(0, t)=\frac{2 \tau_{x}}{\rho_{w}}\left(\sqrt{\frac{t}{\pi \nu}}\right),
$$

and in non-dimensional form Eqn(20) simply is

$$
U\left(0, t^{\prime}\right)=\sqrt{\frac{t^{\prime}}{\pi}}
$$

\section{Case II: Finite depth ocean}

In this case the bottom boundary condition at $z=-h$ where $h$ is the depth of the ocean is given by (8), and the Laplace transform solution of (14) can be assumed in the following manner:

$$
\mathcal{L}\{u\}=A \cosh \left(\sqrt{\frac{s}{\nu}} z\right)+B \sinh \left(\sqrt{\frac{s}{\nu}} z\right) .
$$

Using the sea surface and sea bottom boundary conditions, (22) can be written as

$$
\mathcal{L}\{u\}=\left(\frac{\tau_{x}}{\rho_{w} \sqrt{\nu}}\right) \frac{\sinh \left(\sqrt{\frac{s}{\nu}}(z+h)\right)}{s \sqrt{s} \cosh \left(\sqrt{\frac{s}{\nu}} h\right)} .
$$

The Laplace inverse (see Abramowitz and Stegun [11]) in conjunction with convolution integral, we obtain the solution in non-dimensional form with the independent variables $z^{\prime}=\frac{z}{h}$ and $t^{\prime}=\frac{\nu t}{h^{2}}$ as

$$
\begin{aligned}
U\left(z^{\prime}, t^{\prime}\right)= & \frac{u(z, t)}{\Lambda h} \\
= & \left(1+z^{\prime}\right)-\left(\frac{8}{\pi^{2}}\right) \sum_{n=1}^{\infty} \frac{1}{(2 n-1)^{2}} \cos \left(\frac{(2 n-1) \pi z^{\prime}}{2}\right) \\
& \times \exp \left[-\frac{(2 n-1)^{2} \pi^{2} t^{\prime}}{4}\right] .
\end{aligned}
$$

where $\Lambda=\frac{\tau_{x}}{\rho_{w} \nu}$. This solution is valid in the range $\left(-1 \leq z^{\prime} \leq 0\right)$ and $t^{\prime}>0$ and can be verified easily by using the separation of variables method. The solution at 
the surface $z=0$ is

$$
U\left(0, t^{\prime}\right)=\left[1-\left(\frac{8}{\pi^{2}}\right) \sum_{n=1}^{\infty} \frac{1}{(2 n-1)^{2}} \exp \left(-\frac{(2 n-1)^{2} \pi^{2} t^{\prime}}{4}\right)\right] .
$$

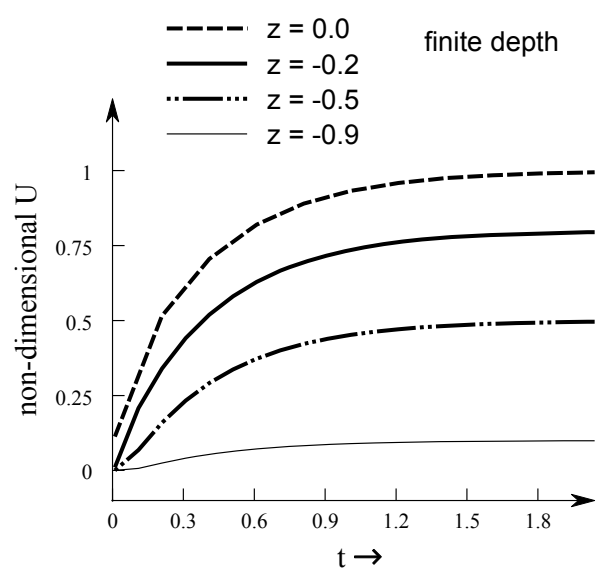

Figure 3: Eulerian currents in finite depth water at various depth.

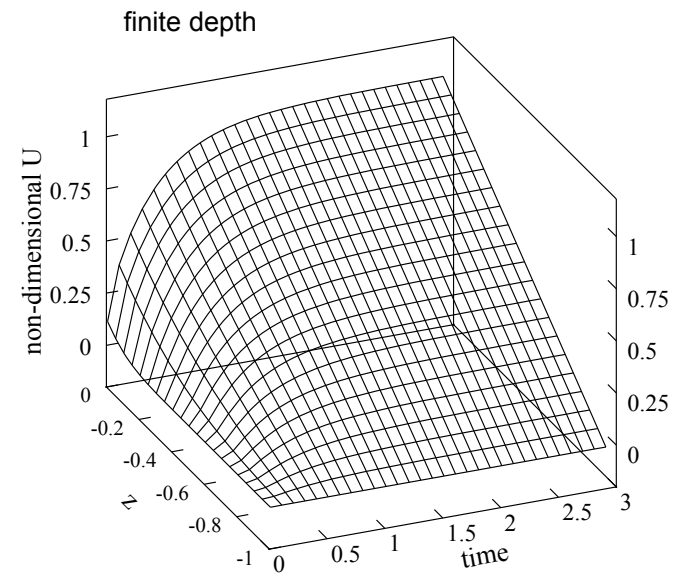

Figure 4: Eulerian currents in finite depth water as a function of $z$ and $t$. 


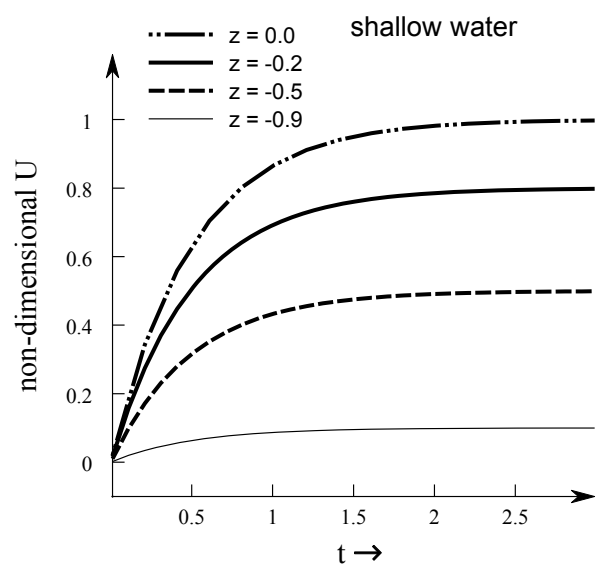

Figure 5: Eulerian currents in shallow water at various depth.

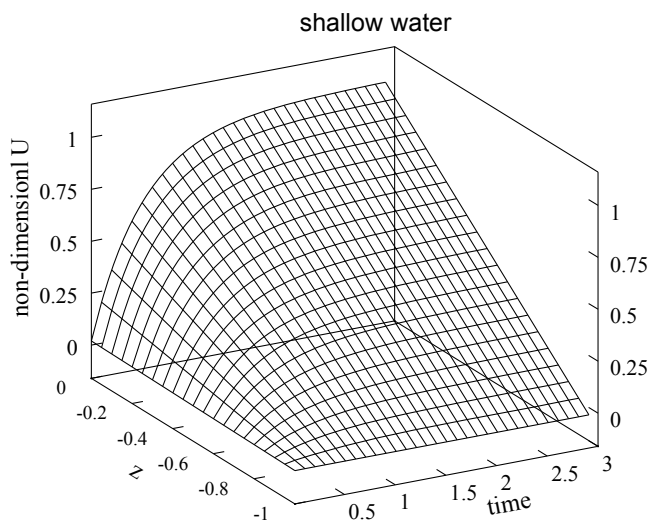

Figure 6: Eulerian currents in shallow water as a function of $z$ and $t$.

\section{Case III: Shallow water ocean}

In this case the Laplace transform solution should be modified by taking into consideration that $h \rightarrow 0$ or $(z+h) \rightarrow 0$. Therefore, the solution simply is (to the order of $\left.\mathrm{O}\left(h^{3}\right)\right)$ :

$$
u(z, t)=\Lambda(z+h)\left[1-\exp \left(-\frac{2 \nu t}{h^{2}}\right)\right]
$$

and at the sea surface $z=0$, this yields

$$
u(0, t)=\Lambda h\left[1-\exp \left(-\frac{2 \nu t}{h^{2}}\right)\right] .
$$


In non-dimensional forms Eqns. (26) and (27) which are valid in the range $\left(-1 \leq z^{\prime} \leq 0\right)$ and $t^{\prime}>0$, can simply be written, respectively, as

$$
\begin{aligned}
U\left(z^{\prime}, t^{\prime}\right) & =\left(1+z^{\prime}\right)\left[1-\exp \left(-2 t^{\prime}\right)\right] \\
U\left(0, t^{\prime}\right) & =\left[1-\exp \left(-2 t^{\prime}\right)\right]
\end{aligned}
$$

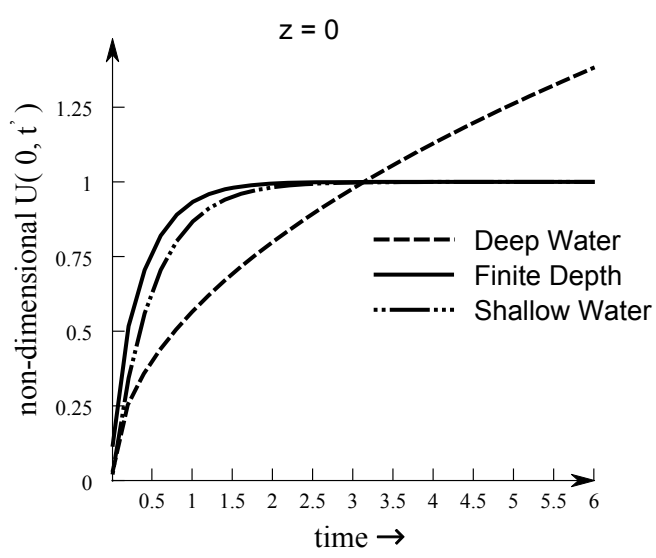

Figure 7: Comparison of Eulerian currents in deep water, finite depth and shallow water at the sea surface.

\section{Results and conclusions}

This is a classical and fundamental theoretical work. The analytical solutions are presented in a rigorous manner to get the elementary understanding of the Eulerian currents in ocean circulations. Mathematical formulations and their solutions are evidently sophisticated. Some graphical solutions are displayed to have a little idea about the Eulerian currents in ocean circulations. The analytical solutions of the Eulerian currents for deep water and finite depth ocean circulations are extremely sophisticated. They are obtained by using very high level mathematical techniques such the Laplace transform method and the separation of variables method. These solutions perfectly satisfied the given governing equation, the boundary conditions and the initial condition. Graphical illustrations in non-dimensional forms are presented in Figures 1-7 to see the behavior of the solutions. Figures 1 and 2 present the solutions for the deep water case, Figures 3 and 4 depict the solutions in finite depth case and Figures 5 and 6 deal with the shallow water case. Comparisons between the Eulerian currents at the sea surface $z=0$ are presented. Figure 7 displays the comparison between the surface currents at the deep water, finite depth water and the shallow water depth. It can be easily seen that they 
comply with the physical situation of the problems. We have carefully checked that the solutions are dimensionally consistent. For further reading about this topic, the scientific papers written by Smith [13] and Ardhuin et al. [14] will be very useful. Detailed mathematical descriptions of this paper are presented in the recent paper by Rahman and Bhatta [15]. The reader can find more information and insights about the mathematical solution techniques in [15].

\section{Acknowledgements}

We are grateful to the Faculty of Computer Science at Dalhousie University for granting its computer facilities. Special thanks are extended to Professor Carlos Brebbia, Director of Wessex Institute of Technology, UK for his constant inspiration and love for this kind of applied physical oceanographic science problem.

\section{References}

[1] Komen, G. J., Hasselmann, S., and Hasselmann, K. (1984). On existence of a fully developed spectrum, J. Phys. Oceanogra., 14, 1271-1285.

[2] Ekman, V. W. (1905). On the influence of the Earth's rotation on ocean currents, Arkiv. F. Matematik. ... ii.

[3] Longuet-Higgins, M. S. (1976). on the nonlinear transfer of energy in the peak of gravity wave spectrum: a simplified model, Proc. Roy. Soc. A347, 311-328.

[4] Hasselmann, K. (1962). On the nonlinear energy transfer in gravity wave spectrum, Part 1: General theory, J. Fluid Mech., 12, 481-500.

[5] Hasselmann, K. (1963). On the nonlinear energy transfer in gravity wave spectrum, Part 2: Conservation theorem, wave-particle analogy, irreversibility, J. Fluid Mech., 15, 273-281. Part 3. ibid, 15, 385-398.

[6] Hasselmann, S., Hasselmann, K. Komen, G. K., Jenssen, P.,Ewing, A. J., and Cardone, V. (1988). The WAM model - A third generation ocean wave prediction model, J. Phys. Oceanogra., 18, 1775-1810.

[7] Rahman, M. (2001). Mathematical modeling of ocean waves, The Ocean Engineering Handbook Edited by F. El-Hawary, CRC Press LLC, Boca Raton, Florida, USA.

[8] Jenkins, A. D. (1989). The use of a wave prediction model for deriving a near surface current model, Dtsch Hydrogr. Z., 42, 134-149.

[9] Komen, G. J., Cavaler, I., Donnelan, M., Hasselmann, K., Hasselmann, S., and Jenssen, P. A. E. M. (1994). Dynamic and Modeling of Ocean Waves, Cambridge University Press, Cambridge.

[10] Perrie, W. and Hu, Y. (1997). Air-ice-ocean momentum exchange, Part II: Ice drift, J. Phys. Oceanogra. 27, 1976-1996.

[11] Abramowitz.M and Stegun, I.A. (1966). Hand Book of Mathematical Functions, The Dover Publications, Inc., New York. 
[12] Rahman, M. (1995). Water Waves: Relating modern theory to advanced engineering applications, Clarendon Press, Oxford.

[13] Smith, J. A., (2006). Wave-current interactions in finite-depth, J. Phys. Oceanogr., 36, 1403-11419.

[14] Ardhuin, F., Marié, L., Rascle, N., Forget, P. and Roland, A., (2009). Observation and estimation of Lagrange, Stokes, and Eulerian currents induced by wind and waves at the sea surface, J. Phys. Oceanogr., 39, 28202838.

[15] Rahman, M. and Bhatta, D., (2014). Computation of Eulerian currents in ocean circulations, International Journal of Applied Mathematics and Engineering Sciences (IJAMES), Volume 8. No. 1, January-June 2014 issue (In Press). 